\title{
ON THE EXISTENCE OF
}

\section{QUANTUM CHARACTERISTIC EXPONENTS}

\author{
R. VILELA MENDES
}
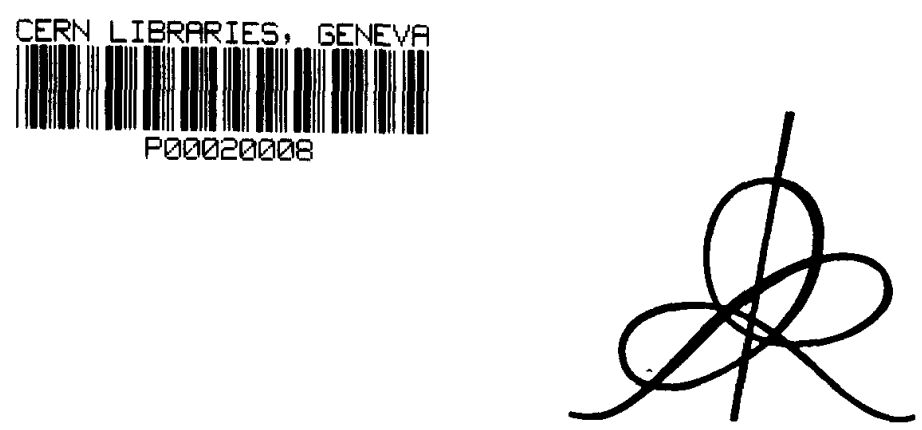

Institut des Hautes Etudes Scientifiques 35 , route de Chartres 91440 - Bures-sur-Yvette (France)

Novembre 1993

IHES/M/93/58 



\title{
On the existence of quantum characteristic exponents
}

\author{
R. Vilela Mendes ${ }^{1}$ \\ Institut des Hautes Etudes Scientifiques \\ 35, route de Chartres \\ 91440 Bures-sur-Yvette, France
}

\begin{abstract}
The Lyapunov characteristic exponents provide a rigorous characterization of the notion of chaos in classical mechanics. Similar quantities have been proposed as a characterization of quantum chaos. In this note we find the conditions under which the existence of quantum characteristic exponents can be proved.
\end{abstract}

${ }^{1}$ Permanent/Mailing address: CFMC - Universidade de Lisboa; Av. Gama Pinto, 2 - 1699 Lisboa Codex, Portugal 
In classical mechanics a dynamical system is said to be chaotic if at least one of its Lyapunov exponents is positive. To attempt a similar characterization of quantum chaos, the notion of quantum sensitive dependence and quantum characteristic exponent was introduced ${ }^{1,2}$, namely

$$
\lambda_{\mathcal{\Lambda},\left\{\phi_{\mathrm{n}}\right\}}=\lim _{\mathbf{k} \rightarrow \infty} \frac{1}{\mathbf{k}} \lim _{\mathbf{n} \rightarrow \infty} \ln \| \operatorname{Re}\left(\mathrm{U}^{\left.\mathbf{k}_{\phi_{\mathrm{n}}}, \mathcal{\Lambda} \mathrm{U}^{\mathrm{k}} \psi\right) \|}\right.
$$

where $\mathrm{U}$ is the time-evolution operator, $\mu$ is a symmetric operator and $\phi_{\mathrm{n}}$ a sequence of functions converging to $\delta_{\mathbf{X}}^{\prime}$.

Ones notices that this expression has a double pointwise nature, depending both on the point $\mathrm{x}$ of $\delta_{\mathrm{x}}^{\prime}$ and the state $\psi$. In Ref. 2 the limit has been shown to exist in some simple models. In the cases that were computed the limit was found to be independent of $\psi$ and to be positive in situations where there is also classical chaos. In this note, by defining an appropriate measure-theoretic setting for the quantum spaces, an existence proof is obtained for the quantum characteristic exponents.

A definition of quantum characteristic exponent of the type of (1.1) is suggested by the form of the classical Lyapunov exponent written in terms of densities $\rho(x)$ in the phase space $M$.

$$
\lambda_{\mathbf{u}, \mathbf{x}}=\lim _{\mathbf{k} \rightarrow \infty} \frac{1}{\mathbf{k}} \ln \left\|-\mathrm{u}^{\mathrm{i}} \mathrm{D}_{\partial_{\mathbf{i}} \delta_{\mathbf{x}}}\left\{\int \mathrm{dy} \mathrm{y} \mathrm{f}^{\mathrm{k}}\left(\psi^{2}(\mathrm{y})\right)\right\}\right\|
$$

$u$ is a vector in the tangent space $T M, f$ is the time-evolution mapping and to emphasize the correspondence with quantum mechanics I have written $\rho(y)=\psi^{2}(y)$. $D_{\partial_{i} \delta_{x}}$ is the Gateaux derivative of the functional $I_{k}=\left\|\int d y f^{k}\left(\psi^{2}(y)\right)\right\|$ in the space of square roots of the densities.

To check that Eq.(2) leads to the usual Lyapunov exponent definition, one uses the properties of the Perron-Frobenius operator ${ }^{[3]}$

$$
\mathrm{f} \rho(\mathrm{y})=\sum_{\mathrm{i}} \rho\left(\mathrm{f}^{-1} \mathrm{y}\right)_{\mathrm{i}} \frac{1}{\left\|\mathrm{Df}\left(\mathrm{f}^{-1} \mathbf{y}\right)_{\mathbf{i}}\right\|}
$$

to change variables and obtain

$$
\lambda_{\mathbf{u}, \mathbf{x}}=\lim _{\mathbf{k} \rightarrow \infty} \frac{1}{\mathbf{k}} \ln \left\|\mathbf{u}^{\mathrm{i}} \partial_{\mathrm{i}}\left\{\mathrm{f}^{\mathrm{k}}(\mathrm{x}) \psi(\mathrm{x})\right\}\right\|
$$


Then, if $\psi(\mathbf{x})$ is a $\mathbf{C}^{\mathbf{1}}$-function

$$
\lambda_{u, x}=\lim _{k \rightarrow \infty} \frac{1}{k} \ln \left\|\mathbf{u}^{i} \partial_{i} f^{k}(x)\right\|
$$

Notice that, except for the regularity conditions, $\lambda_{\mathbf{u}, \mathbf{x}}$ does not depend on the density $\psi^{2}$. Oseledec's theorem asserts that $\lambda_{\mathrm{u}, \mathrm{x}}$ is defined almost everywhere in the support of an f-invariant measure $\mu$ on $M$, being a constant in each ergodic component of $\mu$.

Let $\mathrm{U}^{\mathbf{k}}$ ( $\mathrm{k}$ continuous or discrete) be the unitary operator of quantum time evolution. The quantum analog of Eq.(2) involves the following functional

$$
u^{i} D_{\partial_{i} \delta_{X}}\left(U^{k} \psi, \underset{\sim}{X} U^{k} \psi\right)=2 u^{i} \operatorname{Re}<U^{k} \partial_{i} \delta_{x}, \underset{\sim}{X} U^{k} \psi>
$$

where $U^{-k_{2}} U^{k_{\psi}}$ is assumed to belong for example to the Schwartz space 9 . The quantum space is a space of functions on a manifold $M, \underset{\sim}{X}$ being a coordinate operator in $M$. According to the choice for $M, \underset{\sim}{X}$ may stand either for a space coordinate or a canonical momentum.

To make the limit of (5), when $k \rightarrow \infty$, to be independent of $\psi$ (except for regularity conditions) we will need a norm for the functionals and a U-invariant measure on a space containing $\partial_{i} \delta_{x}$ is also needed to define the domain of existence of the limit. To achieve these goals one constructs a Gelfand triplet

$$
\mathrm{E}^{*} \supset \mathrm{J6} \supset \mathrm{E}
$$

$\mathrm{E}$ being a nuclear space and $\mathrm{E}^{*}$ its dual. It is convenient to obtain $\mathrm{E}$ as a countably normed space, projective limit of a sequence of Hilbert spaces $36_{\mathrm{p}}$.

with ordered norms

$$
E^{*} \supset \cdots \supset{ }^{16}-p \supset \cdots 3_{-1} \supset 3_{6} \supset 3_{1} \supset \cdots \supset 3_{p} \supset \cdots \supset E
$$

$$
\|\cdot\| \leq\|\cdot\|_{1} \leq \cdots \leq\|\cdot\|_{p} \leq \cdots
$$

If the time evolution operator $U$ is a strongly continuous unitary operator on 36 , by Stone's theorem, there is a self-adjoint operator A such that $U=e^{i A}$. Use $A$ to define the sequence of norms by

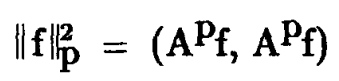

Furthermore $A$ is assumed to be bounded from below and such that there is a $k \in N$ for which $A^{-k}$ is Hilbert-Schmidt. The projective limit space $\mathbf{E}$ is therefore a nuclear space. 
The triplet (6) might be constructed with an operator other than the generator of time evolution. This particular choice, however, insures $U$-invariance of the norms and simplifies the proof below. Nuclearity of $\mathrm{E}$ is important to insure the existence of $\sigma$ additive probability measures on $\mathrm{E}^{*[4]}$.

We may now write the quantum characteristic exponent as follows

$$
\lambda_{\mathbf{u}, \mathbf{x}}=\lim _{\mathbf{k} \rightarrow \infty} \frac{1}{\mathbf{k}} \ln \left\|\mathrm{U}^{-\mathrm{k}} \underset{\sim}{\mathrm{X}} \mathrm{U}^{\mathrm{k}} \mathbf{u}^{\mathrm{i}} \partial_{\mathrm{i}} \delta_{\mathrm{x}}\right\|_{-\ell}
$$

$\|\cdot\|_{-\ell}$ being the norm in $16-\ell$

$$
\|\cdot\|_{-\ell}=\sup _{\phi \in \mathbf{E},\|\phi\|_{\ell} \leq 1} \mid<\cdot, \phi>1
$$

and $\ell$ must greater or equal to the order of the functional $U^{-k}{\underset{\sim}{X}}_{U^{k}} u^{i} \partial_{i} \delta_{x}$. Notice that $U^{k}$, being an element of the infinite dimensional unitary group $U_{\infty}$, has a natural action in $\mathrm{E}^{*[5]}$.

Because $\mathrm{E}$ is a nuclear space, by Minlos theorem, for any continuous functional $\mathrm{C}(\xi) \xi \in \mathrm{E}$ which is positive definite and normalized $(\mathrm{C}(0)=1)$, there is a unique probability measure $\mu$ on $\left(E^{*}, \mathscr{B}\right)$ such that

$$
C(\xi)=\int_{\mathbf{E}^{*}} \mathrm{e}^{\mathrm{i}<\mathrm{x}, \xi>} \mathrm{d} \mu(\mathrm{x})
$$

$\mathscr{B}$ is the Borel $\sigma$-algebra generated by the weak topology (or equivalently the cylinder sets) of $\mathrm{E}^{*}$.

Let $\mu$ be such a probability measure on $\left(E^{*}, \mathfrak{F}\right)$, invariant under the element $U$ of the infinite-dimensional unitary group. Then, existence of the limit in Eq.(7) is a corollary of the following theorem

Theorem: Let $\phi \in 3_{-p},{ }^{36}$ invariant under $U^{-k} \underset{X}{X} U^{k} \quad \forall k$ and $\mu$ a $U$-invariant probability measure in $\left(\mathrm{E}^{*}, \mathfrak{B}\right)$ such that

$$
\int_{E^{*}} \ln \left\|\mathrm{U}^{-k_{2}} \mathrm{U}^{\mathrm{k}_{\phi}}\right\|_{-\mathrm{p}} \mathrm{d} \mu(\phi)
$$

is finite. Then, the limit

$$
\lambda_{\phi}=\lim _{\mathbf{k} \rightarrow \infty} \frac{1}{\mathbf{k}} \ln \left\|U^{-\mathbf{k}} \underset{\sim}{\mathbf{X}} U^{\mathbf{k}}\right\|_{-p}
$$

exists $\mu$-almost everywhere in $36_{-}$p . 
Proof:

$$
\lambda_{\phi}=\lim _{\mathbf{k} \rightarrow \infty} \frac{1}{\mathbf{k}} \ln \left\|\underset{\sim}{\mathbf{X}} \mathrm{U}^{\mathbf{k}} \phi\right\|_{-\mathbf{p}}
$$

follows from the U-invariance of the norms. The functional $g_{k}(\phi): K_{-p} \rightarrow R$ defined by

$$
g_{k}(\phi)=\ln \frac{\left\|\underset{\sim}{X} U^{k}\right\|_{-p}}{\|\underset{\sim}{X}\|_{-p}}=\sum_{j=1}^{k} \ln \frac{\left\|X U^{j} \phi\right\|_{-p}}{\left\|\underset{\sim}{X} U^{j-1}\right\|_{-p}}
$$

is subadditive, $\mathrm{g}_{\mathrm{k}+\mathrm{n}}(\phi) \leq \mathrm{g}_{\mathrm{k}}(\phi)+\mathrm{gn}_{\mathrm{n}}\left(\mathrm{U}^{\mathrm{k}_{\phi}}\right)$. Applying Kingman's theorem ${ }^{[6]}$ the result then follows from $U$-invariance of the measure and the integrability assumptions. $\square$

Provided the stated conditions are satisfied, the theorem asserts the existence $\mu$ almost everywhere of the limit, but not its unicity. That will depend on the ergodic properties of the measure $\mu$ under the one-parameter subgroup of $U_{\infty}$ generated by $U$.

The Gaussian (white noise) measure ${ }^{[5]} \mu_{0}$ given by

$$
\mathrm{C}_{0}(\xi)=\mathrm{e}^{-\|\xi\|^{2}}
$$

is invariant under the whole $U_{\infty}$ group. Therefore quantum characteristic exponents will exist at least in the support of this measure, but the decomposition into ergodic components will depend on the detailed nature of the time-evolution operator $U$.

\section{References}

[1] R. Vilela Mendes; J. Phys. A : Math. Gen. 24 (1991) 4349.

[2] R. Vilela Mendes; Phys. Lett. A171 (1992) 253.

[3] A. Lasota and M. C. Mackey; "Probabilistic properties of deterministic systems", Cambridge Univ. Press, Cambridge 1985.

[4] I. M. Gel'fand and N. Ya. Vilenkin; "Generalized functions", vol. 4, Academic Press, New York 1964.

[5] T. Hida; "Brownian motion", Springer-Verlag, Berlin 1980.

[6] J. F. C. Kingman; J. Roy. Statist. Soc. B30 (1968) 499; Annals of Prob. 1 (1973) 883. 
